\title{
Umweltchemie und Ökotoxikologie in Deutschland
}

\author{
Entwicklungstrend des Faches gemessen an der Publikationsproduktivität
}

\author{
Gerhard Lammel $\cdot$ Martin Scheringer $\cdot$ Klaus Fischer
}

Erhalten: 25. Mai 2009/Akzeptiert: 14. Juni 2009/Online veröffentlicht: 11. Juli 2009

(C) Springer-Verlag 2009

Zusammenfassung Fragestellung Wie gestaltet und ggf. verändert sich die wissenschaftliche Produktivität der Umweltchemie und Ökotoxikologie in Deutschland? So einfach die Frage klingt: Sie ist schwer zu beantworten, denn auf Fachgebieten, die per se interdisziplinär sind, sind Überlappungen zu Nachbargebieten die Regel und Abgrenzungen schwierig, zum Teil unmöglich, weil in der Literatur nicht erkennbar oder nicht erfasst. Umweltchemie und Ökotoxikologie sind oft „drin“, stehen aber nicht „drauf“ - auf den Institutsnamen, den Projekttiteln oder den Zeitschriftentiteln. Bei in hohem Maße interdisziplinären Arbeiten - Tendenz steigend - sind wir Wissenschaftler in manchen Fällen sogar selbst verlegen um eine abgrenzende Zuordnung oder empfänden eine solche als inadäquat oder artifiziell.

Ansatz Der Vorstand der Fachgruppe hat die bibliometrische Analyse anhand von Zeitschriftenstichproben als eine probate Herangehensweise für diese Frage gewählt. Nicht erfasst werden in einer solchen Stichprobe Arbeiten, die in Zeitschriften veröffentlicht wurden, die primär nicht der

G. Lammel

Max-Planck-Institut für Chemie,

J.-J.-Becher-Weg 27, 55128 Mainz, Deutschland

E-Mail: g.lammel@mpic.de

G. Lammel

Research Centre for Environmental Chemistry and Ecotoxicology,

Masaryk University,

Kamenice 3, 62500 Brno, Czech Republic

M. Scheringer

Institut für Chemie- und Bioingenieurwissenschaften, ETH Zürich, HCI G 127, Wolfgang-Pauli-Str. 10, 093 Zürich, Schweiz

E-Mail: scheringer@chem.ethz.ch

K. Fischer $(\bowtie)$

Analytische und Ökologische Chemie, Universität Trier,

Campus II, Behringstr. 21, 54296 Trier, Deutschland

E-Mail: fischerk@uni-trier.de
Umweltchemie oder Ökotoxikologie, sondern einem anderen Fach zugerechnet werden. Von der Analyse erfasst werden insbesondere aber die vielen umweltchemischen und ökotoxikologischen Arbeiten von Wissenschaftlern, deren Arbeitsschwerpunkt in einem anderen Feld liegt und die nur gelegentlich auf unseren Gebieten arbeiten, gleich welche Berufs- und Institutsbezeichnung sie tragen mögen. Diese Fehlerquelle zu vermeiden, erscheint für relative junge Fächer und stark interdisziplinäre Arbeitsgebiete prioritär.

\section{Aktuelle Entwicklung und Randbedingungen wissenschaftlicher Publikationstätigkeit}

Die jährlich erscheinende Anzahl wissenschaftlicher Publikationen nimmt seit Jahren stark zu. In den Jahren 1992, 1997, 2002 und 2007 wurden etwa auf Gebieten der Umwelt- und Geowissenschaften 748, 2543, 3833 und 4167 Originalartikel gezählt (Web of Science - Literaturdatenbank des ISI Web of Knowledge, Thomson Reuters) ${ }^{1}$.

Die Gründe hierfür sind vielfältig: Die Vernetzung der Forschung, sowohl interdisziplinär als auch international, hat deutlich zugenommen. Ein erhöhter Publikationsdruck infolge eines vermehrten Evaluierungswesens bei außeruniversitären und universitären Instituten erzeugt immer weniger Ergebnisse, die von Arbeitsgruppen spät oder gar nicht veröffentlicht werden. Eine gestiegene Bedeutung von Schriftenverzeichnissen als Maßstab der Qualifikation auf der Karriereleiter führt zu effizienteren Publikationsstrategien der Autoren. Nicht notwendigerweise bedeutet eine absolut ge-

\footnotetext{
${ }^{1}$ Dagegen wurde kein klarer Trend für Chemie (ohne Biochemie) verzeichnet: 9828, 10742, 7818 bzw. 7719 Artikel, jedoch für Meteorologie einschließlich atmosphärischer Wissenschaften: 219, 506, 1217 bzw. 1303 Artikel.
} 
stiegene Anzahl von Publikationen in Summe mehr Inhalt als zuvor, oder eine absolut gestiegene Anzahl von Autoren mehr Inhalt im einzelnen Artikel. Nicht von ungefähr wurden Regeln der Mitautorschaft in Codices guter wissenschaftlicher Praxis aufgenommen und wird neuerdings häufig die mit der Autorenzahl umgekehrt gewichtete Anzahl von Artikeln als Maßstab für Publikationstätigkeit herangezogen.

\section{Bibliometrische Stichprobe}

Die Stichprobe umfasst alle Originalartikel und Reviews (aber keine anderen Beiträge, z. B. keine Proceedings, Comments, Editorial Materials) in den drei Jahren 1992, 2002 und 2007 von 15 führenden, ausgewählten Zeitschriften, die der Umweltchemie und Ökotoxikologie zugerechnet werden können (Tabelle 1a), sowie in 13 führenden, ausgewählten Zeitschriften der gleichen Jahre, die der analytischen Chemie zugerechnet werden können (Tabelle 1b). Eine Zeitschrift, das International Journal of Environmental Analytical Chemistry, wird hierbei beiden Gebieten zugerechnet. Eine weitere Zeitschrift, die Environmental Science and Pollution Research, erschien im Jahre 1992 noch nicht. Die Zeitschriften, die der Umweltchemie und Ökotoxikologie zugerechnet werden, wurden zusätzlich für die Jahre 2006 und 2008 ausgewertet. Recherchiert wurde ausschließlich in der führenden Literaturdatenbank ISI-Web of Science von Thomson Scientific (http://www.isiknowledge.com/WOS). Erfasst wurden Beiträge mit Beteiligung von Autoren, die eine deutsche Institutsadresse führen und solche, die eine

Tabelle 1 Bibliometrische Daten ausgewählter Zeitschriften (a) der Umweltchemie und Ökotoxikologie und (b) der analytischen Chemie der Jahrgänge 1992/2002/2007

\begin{tabular}{|c|c|c|c|c|}
\hline \multicolumn{5}{|l|}{ a. Umweltchemie und Ökotoxikologie } \\
\hline \multirow[t]{2}{*}{ Journal } & & Beiträge gesamt & Beiträge BRD & Beiträge UK \\
\hline & Jahr & $1992 / 2002 / 2007$ & 1992/2002/2007 & $1992 / 2002 / 2007$ \\
\hline \multirow[t]{2}{*}{ Atmospheric Environment } & abs. & $291 / 522 / 729$ & $19 / 42 / 59$ & $45 / 50 / 73$ \\
\hline & $\%$ & & $6,5 / 8,0 / 8,1$ & $15,5 / 9,6 / 10,0$ \\
\hline \multirow{2}{*}{$\begin{array}{l}\text { Bulletin of Environmental Contamination \& } \\
\text { Toxicology }\end{array}$} & abs. & $276 / 253 / 248$ & $2 / 6 / 1$ & $3 / 9 / 5$ \\
\hline & $\%$ & & $0,7 / 2,4 / 0,4$ & $1,1 / 3,6 / 2,0$ \\
\hline \multirow[t]{2}{*}{ Chemosphere } & abs. & $276 / 475 / 1052$ & $35 / 69 / 72$ & $15 / 22 / 56$ \\
\hline & $\%$ & & $12,7 / 14,5 / 6,8$ & $5,4 / 4,6 / 5,3$ \\
\hline \multirow[t]{2}{*}{ Ecotoxicology \& Environ. Safety } & abs. & $64 / 129 / 156$ & $4 / 8 / 8$ & $4 / 9 / 9$ \\
\hline & $\%$ & & $6,3 / 6,2 / 5,1$ & $6,3 / 7,0 / 5,8$ \\
\hline \multirow[t]{2}{*}{ Environmental Health Persp. } & abs. & $61 / 272 / 243$ & $0 / 5 / 12$ & $3 / 12 / 9$ \\
\hline & $\%$ & & $0,0 / 1,8 / 4,9$ & $4,9 / 4,4 / 3,7$ \\
\hline \multirow[t]{2}{*}{ Environmental Pollution } & abs. & $78 / 232 / 301$ & $0 / 10 / 22$ & $13 / 29 / 47$ \\
\hline & $\%$ & & $0,0 / 4,3 / 7,3$ & $16,7 / 12,5 / 15,6$ \\
\hline \multirow[t]{2}{*}{ Environmental Science \& Pollut Res } & abs. & $-/ 92 / 63$ & $-/ 39 / 22$ & $-/ 7 / 1$ \\
\hline & $\%$ & & $-/ 42,4 / 34,9$ & $-/ 7,6 / 1,6$ \\
\hline \multirow[t]{2}{*}{ Environmental Science \& Technology } & abs. & $351 / 791 / 1194$ & $12 / 64 / 66$ & $6 / 49 / 88$ \\
\hline & $\%$ & & $3,4 / 8,1 / 5,5$ & $1,7 / 6,2 / 7,4$ \\
\hline \multirow[t]{2}{*}{ Environmental Toxicology \& Chemistry } & abs. & $119 / 316 / 301$ & $0 / 13 / 13$ & $3 / 25 / 17$ \\
\hline & $\%$ & & $0,0 / 4,1 / 4,3$ & $2,5 / 7,9 / 5,6$ \\
\hline \multirow[t]{2}{*}{ Geophysical Research Letters } & abs. & $615 / 1214 / 1478$ & $34 / 122 / 151$ & $39 / 121 / 207$ \\
\hline & $\%$ & & $5,5 / 10,0 / 10,2$ & $6,3 / 10,0 / 14,0$ \\
\hline \multirow[t]{2}{*}{ Int. J. Environ. Analytical Chemistry } & abs. & $63 / 64 / 77$ & $13 / 4 / 5$ & $5 / 3 / 6$ \\
\hline & $\%$ & & $20,6 / 6,3 / 6,5$ & $7,9 / 4,7 / 7,8$ \\
\hline \multirow[t]{2}{*}{ Journal of Atmospheric Chemistry } & abs. & $64 / 42 / 46$ & $18 / 22 / 10$ & $3 / 4 / 5$ \\
\hline & $\%$ & & $28,1 / 52,4 / 21,7$ & $4,7 / 9,5 / 10,9$ \\
\hline \multirow[t]{2}{*}{ Marine Pollut. Bulletin } & abs. & $100 / 162 / 228$ & $2 / 4 / 10$ & $18 / 15 / 47$ \\
\hline & $\%$ & & $2,0 / 2,5 / 4,4$ & $18,0 / 9,3 / 20,6$ \\
\hline \multirow[t]{2}{*}{ The Science of the Total Environ. } & abs. & $244 / 307 / 564$ & $19 / 22 / 32$ & $40 / 67 / 56$ \\
\hline & $\%$ & & $7,8 / 7,2 / 5,7$ & $16,4 / 21,8 / 9,9$ \\
\hline \multirow[t]{2}{*}{ Water, Air and Soil Pollution } & abs. & $174 / 206 / 279$ & $13 / 14 / 15$ & $8 / 9 / 16$ \\
\hline & $\%$ & & $7,5 / 6,8 / 5,4$ & $4,6 / 4,4 / 5,7$ \\
\hline \multirow[t]{2}{*}{ Summe (a) } & $a b s$. & $2776 / 5077 / 6959$ & $171 / 444 / 498$ & $205 / 431 / 642$ \\
\hline & $\%$ & & $6,2 / 8,7 / 7,2$ & $7,4 / 8,5 / 9,2$ \\
\hline
\end{tabular}




\begin{tabular}{|c|c|c|c|c|}
\hline \multicolumn{5}{|l|}{ b. Analytische Chemie } \\
\hline \multirow[t]{2}{*}{ Journal } & & Beiträge gesamt & Beiträge BRD & Beiträge UK \\
\hline & Jahr & 1992/2002/2007 & 1992/2002/2007 & $1992 / 2002 / 2007$ \\
\hline \multirow[t]{2}{*}{ Analysis } & abs. & $48 / 63 / 60$ & $0 / 0 / 1$ & $22 / 19 / 34$ \\
\hline & $\%$ & & $0,0 / 0,0 / 1,7$ & $45,8 / 30,2 / 56,7$ \\
\hline \multirow[t]{2}{*}{ Analyst } & abs. & $255 / 296 / 156$ & 7/10/9 & $54 / 75 / 41$ \\
\hline & $\%$ & & $2,7 / 3,4 / 5,8$ & $21,2 / 25,3 / 26,3$ \\
\hline \multirow[t]{2}{*}{ Analytica Chimica Acta } & abs. & $498 / 598 / 838$ & $24 / 26 / 22$ & $35 / 28 / 33$ \\
\hline & $\%$ & & $4,8 / 4,3 / 2,6$ & $7,0 / 4,7 / 3,9$ \\
\hline \multirow[t]{2}{*}{ Analytical Chemistry } & abs. & $540 / 904 / 1176$ & $16 / 51 / 88$ & $13 / 48 / 106$ \\
\hline & $\%$ & & $3,0 / 5,6 / 7,5$ & $2,4 / 5,3 / 9,0$ \\
\hline \multirow[t]{2}{*}{ Analytical Letters } & abs. & $178 / 194 / 258$ & $6 / 3 / 7$ & $3 / 7 / 6$ \\
\hline & $\%$ & & $3,4 / 1,5 / 2,7$ & $1,7 / 3,6 / 2,3$ \\
\hline \multirow{2}{*}{$\begin{array}{l}\text { Fresenius J. Analytical Chemistry/Analytical } \\
\text { Bioanalytical Chemistry }{ }^{\mathrm{a}}\end{array}$} & abs. & $261 / 370 / 582$ & $107 / 67 / 93$ & $6 / 4 / 33$ \\
\hline & $\%$ & & $41,0 / 18,1 / 16,0$ & $2,3 / 1,1 / 5,7$ \\
\hline \multirow[t]{2}{*}{ Int. J. Environ. Analytical Chemistry } & abs. & $63 / 64 / 77$ & $13 / 4 / 5$ & $5 / 3 / 6$ \\
\hline & $\%$ & & $20,6 / 6,3 / 6,5$ & $7,9 / 4,7 / 7,8$ \\
\hline \multirow[t]{2}{*}{ Journal of Chromatogr } & abs. & $915 / 1162 / 1689$ & $66 / 95 / 88$ & $42 / 49 / 75$ \\
\hline & $\%$ & & $7,2 / 8,2 / 5,2$ & $4,6 / 4,2 / 4,4$ \\
\hline \multirow[t]{2}{*}{ J. Radioanalytical Nuclear Chemistry ${ }^{\mathrm{b}}$} & abs. & $356 / 347 / 345$ & $8 / 17 / 17$ & $9 / 1 / 10$ \\
\hline & $\%$ & & $2,2 / 4,9 / 4,9$ & $2,5 / 0,3 / 2,9$ \\
\hline \multirow[t]{2}{*}{ Nuclear Instruments Methods Physics ${ }^{\mathrm{b}}$} & abs. & $505 / 755 / 520$ & $80 / 132 / 75$ & $45 / 58 / 52$ \\
\hline & $\%$ & & $15,8 / 17,5 / 14,4$ & $8,9 / 7,7 / 10,0$ \\
\hline \multirow[t]{2}{*}{ Spectrochim. Acta } & abs. & $273 / 362 / 688$ & $21 / 35 / 25$ & $32 / 16 / 3$ \\
\hline & $\%$ & & $7,7 / 9,7 / 3,6$ & $11,7 / 4,4 / 0,4$ \\
\hline \multirow[t]{2}{*}{ Talanta } & abs. & $246 / 349 / 737$ & $5 / 7 / 12$ & $15 / 12 / 9$ \\
\hline & $\%$ & & $2,0 / 2,0 / 1,6$ & $6,1 / 3,4 / 1,2$ \\
\hline \multirow[t]{2}{*}{ Summe (b) } & $a b s$. & $4075 / 5464 / 7049$ & $340 / 447 / 437$ & $276 / 320 / 402$ \\
\hline & $\%$ & & $8,3 / 8,2 / 6,2$ & $6,8 / 5,9 / 5,7$ \\
\hline
\end{tabular}

a $\mathrm{ABC}$ ist aus dem FJAC hervorgegangen.

${ }^{\mathrm{b}}$ Ausschließlich Beiträge aus der analytischen Chemie ausgewertet.

Institutsadresse in einem Vergleichsland, dem Vereinigten Königreich (UK) von Großbritannien und Nord Irland, führen. Hierbei werden alle, nicht nur die Erst- oder Korrespondenzautoren, erfasst.

\section{Ergebnisse}

Gegenüber 1992 war es 2007 den deutschen Forschungsinstituten gelungen, ihren Anteil am internationalen Publikationsvolumen im Bereich „Umweltchemie und Ökotoxikologie“ von $6,2 \%$ auf $7,2 \%$ zu steigern. Wegen des gewaltigen Anstiegs des weltweiten „Publikationsausstoßes“ in diesem Zeitraum (von ca. 2800 auf ca. 7000 Artikel in der analysierten Zeitschriftenstichprobe) war für diesen kleinen prozentualen Gewinn aber fast eine Verdreifachung der Artikelproduktion von 171 auf 498 notwendig. Unterschiedliche Zeitschriften trugen hierzu mit unterschiedlichen Trends bei (Tabelle 1a, Abb. 1a). Bezogen auf das Jahr 2002 war im Jahr 2007 ebenfalls noch ein absoluter Anstieg zu verzeichnen (Zuwachs von 444 auf 498 Artikel). Dieser Anstieg war jedoch in den relativen Zahlen nicht mehr sichtbar: Der Anteil von Artikeln mit deutscher Beteiligung war 2002 höher als 2007, er ging ab 2002 von $8,7 \%$ auf 7,2\% zurück.

Weil nur wenige Jahre analysiert wurden, kennen wir das Jahr der maximalen Publikationsaktivität der deutschen Umweltforschung und Ökotoxikologie nicht, erfahren aber immerhin, dass sie im Zeitraum 1992-2007 durch (mindestens) ein Maximum ging. Dieses ist signifikant, denn die Unterschiede zwischen aufeinanderfolgenden Jahren sind wohl geringer, wie die Analyse der Jahre 2006-2008 zeigt: In diesen Jahren summiert sich die absolute (in Klammern: relative) Publikationstätigkeit in der genannten Jahresabfolge zu 464 (6,6\%), 498 (7,2\%) und 521 Artikeln (7,2\%). Die Publikationsleistung konnte also im Jahre 2008 nur noch absolut, nicht mehr relativ gesteigert werden.

Wie stellt sich dieser Verlauf dar im Vergleich mit (a) einem anderen Fach und (b) einem anderen Land? Denn angesichts von sich rasch veränderndem Publikationswesen und sich rasch verändernder Publikationskultur sind für unsere Betrachtung vor allem komparative Veränderungen relevant. 

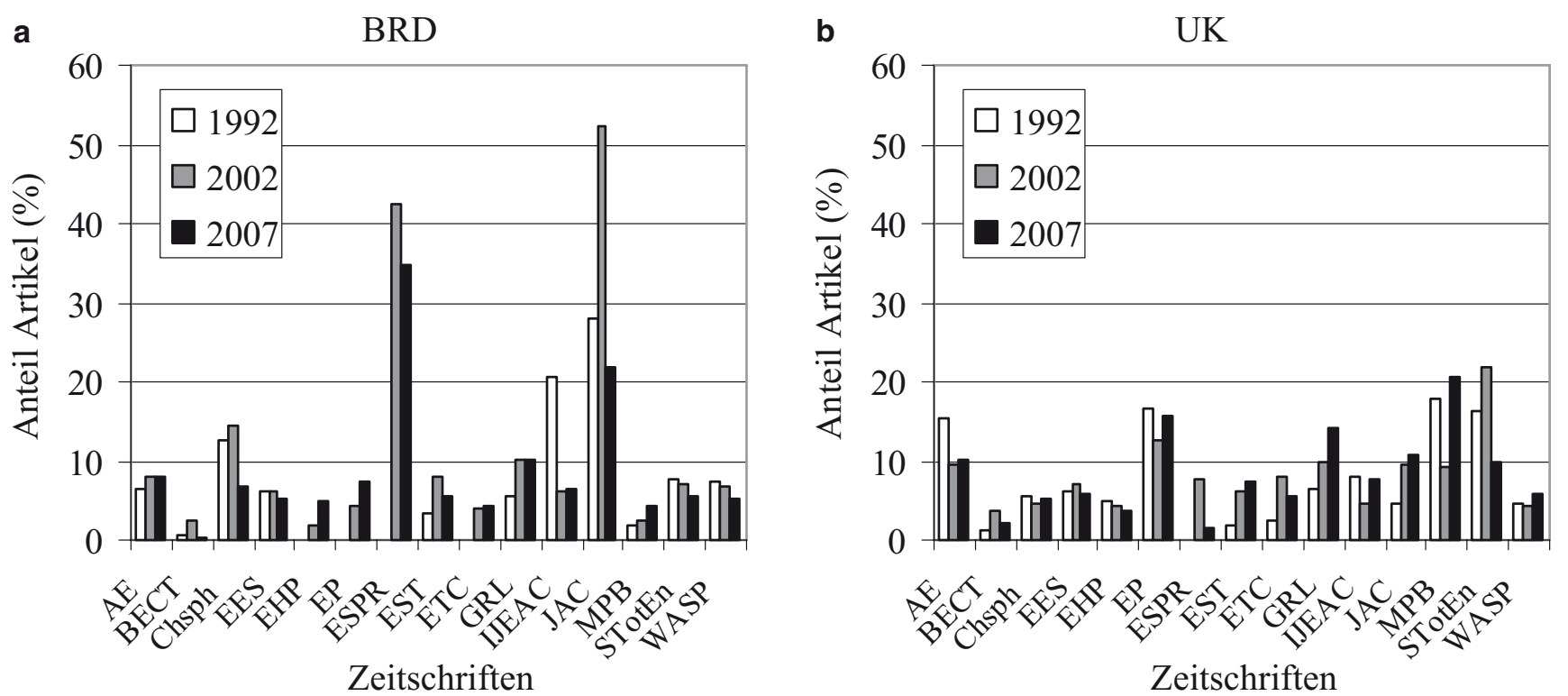

Abb. 1a,b Anteile an Originalartikeln und Reviews in ausgewählten Zeitschriften² der Umweltchemie und Ökotoxikologie der Jahrgänge 1992, 2002 und 2007 mit Beteiligung von deutschen (a) und britischen ${ }^{3}$ Instituten (b)

\section{1 ... im Vergleichsfach Analytische Chemie}

Für die Analytische Chemie wurde eine ähnlich große Stichprobe für die selben Jahre ausgewertet (zwölf maßgebliche Zeitschriften, ca. 4000 Artikel für 1992 und ca. 7000 Artikel für 2007; Tabelle 1b). Die deutschen Institute steigerten in diesem Zeitraum die Anzahl ihrer Publikationen um 29\%, d.h. von 353 auf 442 Artikel, was nicht genügte, um eine deutliche Abnahme ihres Anteils an der weltweiten Publikationstätigkeit in diesem Fach zu verhindern, nämlich von 8,5\% im Jahr 1992 auf 6,2\% im Jahr 2007. Bis 2002 konnte die Position immerhin gehalten werden (Veröffentlichung von 447 Artikeln im Jahr 2002), um dann rasch abzufallen (von 8,2\% auf 6,2\%; Abb. 2b), d.h., das Zurückfallen im internationalen Vergleich geschah praktisch vollständig im Zeitraum 2002-2007.

\section{2 ... im Vergleichsland Vereinigtes Königreich}

Institute im UK konnten die Anzahl ihrer Publikationen in Umweltchemie und Ökotoxikologie im betrachteten Zeitraum etwas mehr als verdreifachen, wodurch sie ihren Vor-

\footnotetext{
${ }^{2} \mathrm{AE}=$ Atmospheric Environment, BECT $=$ Bulletin of Environmental Contamination \& Toxicology, $\mathrm{Chsph}=$ Chemosphere, EES $=$ Ecotoxicology Environmental Safety, EHP = Environmental Health Perspectives, EP = Environmental Pollution, ESPR $=$ Environmental Science \& Pollution Research, EST = Environmental Science \& Technology, ETC $=$ Environmental Toxicology \& Chemistry, GRL $=$ Geophysical Research Letters, IJEAC = International Journal of Environmental Analytical Chemistry, JAC $=$ Journal of Atmospheric Chemistry, MPB $=$ Marine Pollution Bulletin, STotEn $=$ The Science of the Total Environment, WASP $=$ Water, Air and Soil Pollution.

${ }^{3}$ Einschließlich Nord-Irland.
}

sprung im Jahr 1992 von 7,4\% (gegenüber 6,2\% aus der BRD) auf 9,2\% (gegenüber 7,2\% aus der BRD) im Jahr 2007 ausbauen konnten (siehe Tabelle 1a, Abb. 1b). Im Jahr 2002 wurde ein Publikationsanteil von 8,5\% erreicht - in diesem Jahr lag der deutsche Anteil leicht höher (bei 8,7\%, Abb. 2a).

Nicht berücksichtigt wurde in der Stichprobe die Zeitschrift Atmospheric Chemistry and Physics (ACP), die seit 2000 erscheint und aufgrund einer sehr großen Artikelzahl (948 im Jahr 2007) und einer starken deutschen Verankerung das Ergebnis des Ländervergleiches stark beeinflussen würde. Schließt man ACP in die Stichprobe mit ein, ergibt sich für 2007 tatsächlich ein etwas anderes Bild: Der Vorsprung britischer Institute wäre dann mit 9,8\% gegenüber $8,8 \%$ Publikationsanteil deutscher Institute zu beziffern und damit etwas geringer. Der Anteil der Publikationen in Umweltchemie und Ökotoxikologie, die aus der Atmosphärenforschung insgesamt kommen, lag in den untersuchten Jahren in der BRD bei $50-59 \%$ und im UK bei $47-50 \%{ }^{4}$

Diese Angaben sind, weil die Zuordnung von Zeitschriften zu den zu Fachgebieten unscharf ist, mit einer zusätzlichen Unsicherheit behaftet.

In der Analytischen Chemie konnten britische Institute (einschl. Nordirland) ihre Publikationstätigkeit im 15-Jahreszeitraum um 46\% erhöhen (von 281 Artikeln 1992 auf

\footnotetext{
${ }^{4}$ Hierzu wurden sieben zusätzliche Zeitschriften erfasst, darunter vier, die der Atmosphärenforschung zugerechnet werden, nämlich Atmospheric Chemistry and Physics, Fresenius Environmental Bulletin, Journal of Aerosol Science, Journal of Environmental Monitoring, Journal of Geophysical Research Atmosphere, Tellus und Water Research.
} 

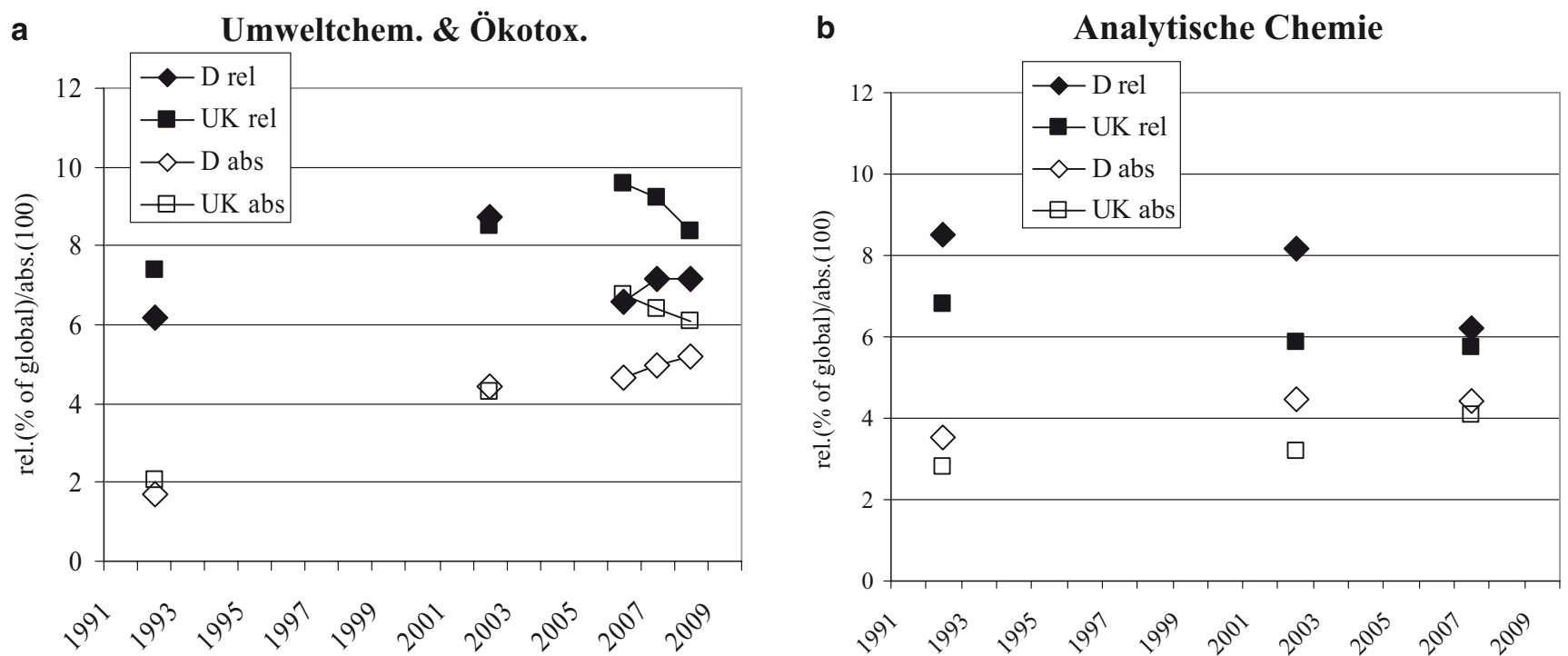

Abb. 2a,b Trends der Anteile an der weltweiten Publikationsleistung (in Prozent) und der Jahrespublikationszahlen (abs. $\times 100)$ der deutschen und britischen ${ }^{5}$ Instituten zuzurechnenden Veröffentlichungen in ausgewählten Zeitschriften der Umweltchemie und Ökotoxikologie (a) und der Analytischen Chemie (b)

408 im Jahr 2007). Dennoch ging damit ihr Anteil am gesamten einschlägigen Publikationsvolumen von $6,8 \%$ auf $5,7 \%$ zurück, wobei der Rückgang weniger stark ausgeprägt war als bei den deutschen Instituten. Ab dem Jahr 2002 näherten sich die Publikationszahlen britischer und deutscher Institute an.

\section{Schlussfolgerungen}

Der Anteil von Artikeln mit deutscher Beteiligung am internationalen Publikationsvolumen in den Gebieten Umweltchemie und Ökotoxikologie stieg von 1992 bis 2002 an und fiel von 2002 bis $2007 \mathrm{ab}$. Wegen der weltweit raschen Zunahme der wissenschaftlichen Publikationstätigkeit sind starke absolute Zuwächse erforderlich, um früher erreichte Anteile zu halten. Noch etwas mehr publizierten Institute

${ }^{5}$ Einschließlich Nord-Irland. aus einem vergleichbar forschungsstarken Land, dem UK, in den Jahren 1992 und 2007 (geringer Unterschied zu deutschen Instituten im Jahr 2002). Ihr Anteil wuchs bis 2006 und fällt seither ebenfalls ab. In einem Vergleichsfach, der Analytischen Chemie, waren die Zuwächse dagegen zu schwach, um die relativen Anteile im Vergleichszeitraum über das Jahr 2002 hinaus halten zu können.

Insgesamt deuten die Ergebnisse darauf hin, dass die deutsche Publikationsleistung im Gebiet Umweltchemie und Ökotoxikologie, gemessen an der weltweiten Zahl der Publikationen, in den vergangenen Jahren zurückgegangen ist. Wir halten es für geboten, einer solchen Entwicklung durch eine verbesserte Förderung von Forschung und Lehre in Umweltchemie und Ökotoxikologie entgegenzuwirken.

\section{Literatur}

http://www.isiknowledge.com/WOS 